\title{
Optimization of Multiple Coils Immersed in a Conducting Liquid for Half-Hemisphere or Whole-Brain Deep Transcranial Magnetic Stimulation: A Simulation Study
}

\author{
Sónia C.P. Sousa-IEEE EMBS Student Member, Jorge Almeida, Ricardo Salvador, João Silvestre, \\ Hugo Simões-IEEE Student Member, and Paulo Crespo-IEEE EMBS Member
}

\begin{abstract}
Transcranial magnetic stimulation (TMS) was proposed in 1985. Nevertheless, its wider use in the treatment of several neurologic diseases has been hindered by its inability to stimulate deep-brain regions. This is mainly due to the physical limiting effect arising from the presence of surface discontinuities, particularly between the scalp and air. Here, we present the optimization of a system of large multiple coils for whole-brain and half-hemisphere deep TMS, termed orthogonal configuration. COMSOL ${ }^{\circledR}$-based simulations show that the system is capable of reaching the very center of a spherical brain phantom with $58 \%$ induction relative to surface maximum. Such penetration capability surpasses to the best of our knowledge that of existing state of the art TMS systems. This induction capability strongly relies on the immersion of the stimulating coils and part of the head of the patient in a conducting liquid (e.g. simple saline solution). We show the impact of the presence of this surrounding conducting liquid by comparing the performance of our system with and without such liquid. In addition, we also compare the performance of the proposed coil with that of a circular coil, a figure-eight coil, and the H-coil. Finally, in addition to its whole-brain stimulation capability (e.g. potentially useful for prophylaxis of epileptic patients) the system is also able to stimulate mainly one brain hemisphere, which may be useful in stroke rehabilitation, among other applications.
\end{abstract}

\section{INTRODUCTION}

TMS is a medical non-invasive technique capable of treating neuropsychiatric disorders, such as depression [1, 2] (approved by the United States Food Drug and Administration in 2008). In addition, TMS has been considered to be a promising therapeutic method not only for others neuropsychiatric disorders [3] as well as neurological diseases [4-12]. The coils of the TMS device deliver a

Research supported by an INOV.C grant co-funded by QREN, under the Mais Centro Program and the European Regional Development Fund, and Fundação para a Ciência e a Tecnologia, Lisbon, Portugal.

S.C.P. Sousa, J. Silvestre, H. Simões, and P. Crespo are with the LIP - Laboratório de Instrumentação e Física Experimental de Partículas, University of Coimbra, 3004-516 Coimbra, Portugal (phone: +351-239410-662; fax: +351-239-822-358; e-mail: sonia.sousa@coimbra.lip.pt, silvestr@isec.pt, hugo.simoes@ coimbra.lip.pt, crespo@lip.pt).

S.C.P. Sousa, H. Simões, and P. Crespo are also with the Physics Department, University of Coimbra, 3004-516 Coimbra, Portugal.

J. Silvestre is also with ISEC- Instituto Superior de Engenharia de Coimbra, Quinta da Nora, 3030-199 Coimbra, Portugal.

J. Almeida is with Proaction Lab, Faculdade de Psicologia e Ciências da Educação, University of Coimbra, 3001-802 Coimbra, Portugal (e-mail: jorgealmeida@fpce.uc.pt).

R.N. Salvador is with IBEB- Instituto de Biofísica e Engenharia Biomédica, University of Lisbon, 1749-016, Lisbon, Portugal (e-mail: rnsalvador@fc.ul.pt) changing electrical current that produces a time-varying magnetic field perpendicular to the current passing through the coils. This magnetic field passes through the soft tissues of the head and the skull to reach the conductive brain tissue where it induces an electrical current (Fig. 1) able to modulate the neural transmembrane potentials and, therefore, neural activity. Magnetic pulses can be applied individually (single-pulse TMS) or in the form of trains of stimuli (repetitive TMS, rTMS). The latter can be divided into low (1-2 Hz) and high $(5-20 \mathrm{~Hz})$ frequency rTMS with inhibitory and excitatory effects, respectively [13].

\section{A. Putative applications of deep-brain TMS}

Several putative applications of rTMS in therapy have been described in the literature. Numerous studies have sought to identify the key areas involved in the pathogenesis of several diseases, e.g. depression. Several studies employing different experimental approaches in humans have provided convergent and compelling evidence that ventromedial (vmPFC) and dorsolateral (dlPFC) regions of the prefrontal cortex are key neural substrates underlying depression, with hypoactivity in dlPFC but hyperactivity in vmPFC [1]. High-frequency stimulation of the dlPFC is the conventional non-invasive treatment of depression beyond drugs. The most potent forms of neuromodulation (i.e. electroconvulsive therapy and deep-brain stimulation, DBS) are typically directed at non-dlPFC targets, some of which lie deep in the cranial vault, which are inaccessible with standard TMS equipment. The vmPFC region lies at a depth of $7 \mathrm{~cm}$, too deep for stimulation with conventional coils.

Another interesting putative application is in epilepsy. The latter is categorized based on various recurrent attacks, and its representation lies in the brain and progressively spreads from seizure origin to different areas, and even the whole brain. The temporal lobe is the most prevalent site for the generation of epileptic seizures. Today, anticonvulsive medications are largely used to treat epilepsy but about $20 \%$ of epileptics are drug resistant. In such cases, surgery may be

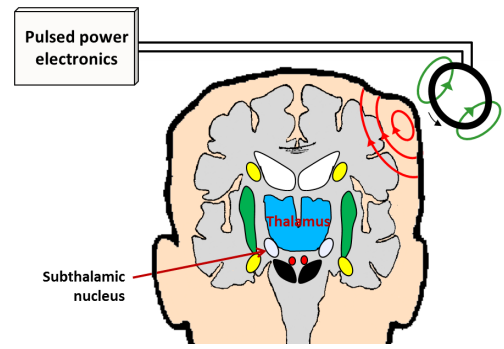

Figure 1. Scheme showing a circular coil current and induced electric field lines for a conventional TMS device. 
used, although in several cases it causes irreversible side effects, leading to neuronal damage [4]. The effects of lowfrequency rTMS (LF-rTMS) remain after the end of stimulation and are N-methyl-D-aspartate (NMDA)-receptor dependent, thus indicating that long term depression (LTD)inducing protocols might have antiepileptic properties. This method thus seems to be promising for prophylaxis and treatment of epileptic seizures. The antipileptic effects of TMS have been investigated in a series of open-label studies, single-case reports, and controlled studies, which have shown that LF-rTMS reduces seizure frequency and epileptic discharges in epilepsy patients [5]. DBS is used for treatment of Parkinson's disease (PD), and targets are subthalamic nucleus (Fig.1), globus pallidus internus, and ventral intermediate nucleus of the thalamus [6], all located in deep brain regions. High-frequency deep-brain rTMS applied over the motor and prefrontal cortices with a Hesed coil (H-coil) has shown to be safe in PD and motor symptoms significantly improved after stimulation [7]. TMS has also been investigated as a potential tool for modulating motor recovery in stroke. Cortical reorganization after stroke produces an imbalance of interhemispheric inhibition, which may be reduced by inhibitory or excitatory rTMS to the contralesional or ipsilesional hemisphere, respectively [8]. A single session of excitatory deep-brain rTMS over the right inferior frontal gyrus with the H-coil significantly improves naming performance in right-handed chronic post stroke aphasic patients. This stimulation induced facilitation of intra- and inter-hemispheric language networks by activating axons in the cortex and subcortical white matter tracts as well [9]. Other fields of clinical application with ongoing research and development have also been identified, e.g. sleep disorders [10], pain management [11], schizophrenia [3], and Alzheimer's disease [12], among others.

\section{B. State of the art}

The two most common coil geometries available are the circular (Fig.1) and figure-eight coils. Circular coils induce a non-focal ring-shaped electric field maximum potentially stimulating brain regions under the coil perimeter. Figureeight coils consist of a pair of adjacent circular loops with current flow in opposite directions, producing a relatively focal electric field maximum under the center of the coil where the two loops meet [14].

There has been substantial interest in direct, non-invasive stimulation of brain regions deeper than the superficial cortex. However, the design of TMS coils to stimulate such deep brain targets is limited by the rapid attenuation of the electric field in depth. The H-coil [15] has been proposed for stimulating deeper cerebral regions. To the best of our knowledge it represents the state of the art of clinical deepbrain TMS. The H-coil has complex winding patterns that result in slower electric field attenuation with depth, at the expense of reduced focality. Nevertheless, there is not any coil able to produce an effective field at the center of the brain. Therefore, the major challenge in deep-brain TMS is to build a device which stimulates deeper brain structures, specifically the target regions for the treatment of several of the aforementioned diseases. The optimized orthogonal configuration presented here meets this challenge, being able to stimulate at a brain depth of $10 \mathrm{~cm}$ with $58 \%$ strength relative to surface maximum. This system can also achieve $75 \%$ and $68 \%$ relative induction (RI) at a brain depth of 6 and $7 \mathrm{~cm}$, respectively (Fig. 2). Comparatively, the H-coil reaches $47 \% \mathrm{RI}$ at $7 \mathrm{~cm}$ brain depth $[15,16]$.

\section{THE IMPACT OF USING A CONDUCTING LIQUID}

In Fig. 3 the impact of using a conducting liquid surrounding a circular coil and a spherical head model can be seen. The cerebrum is surrounded by three principal layers, specifically CSF, skull, and skin. Their electrical conductivities are $0.33,1.79,0.008$, and $0.33 \mathrm{~S} / \mathrm{m}$, respectively [17]. In Fig. 4 the performance of the figure-

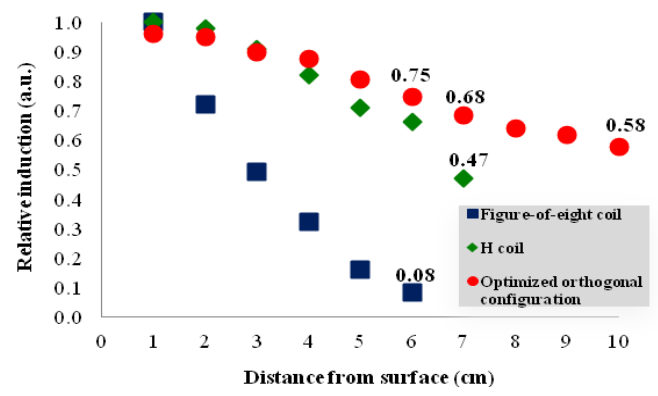

Figure 2. Induction ratio (IR) for two modern coils for superficial (figureeight coil) and deep-brain TMS (H-coil). The optimized orthogonal configuration presented here reaches a depth of $10 \mathrm{~cm}$, for the first time, with $58 \%$ induction relative to surface maximum.

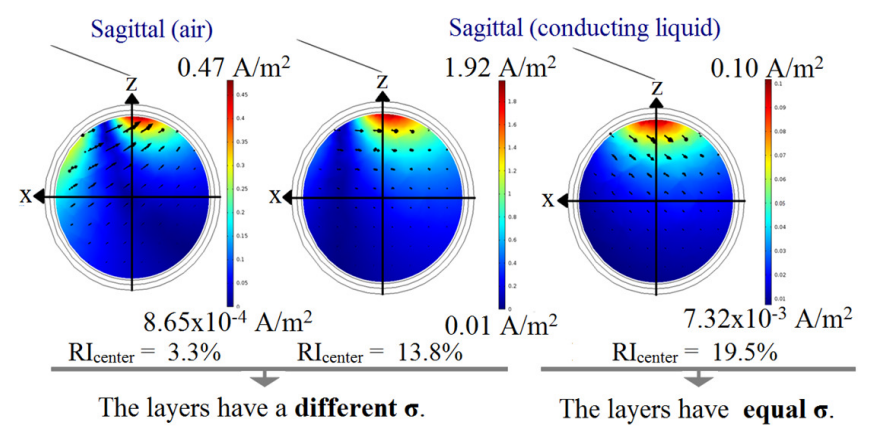

Figure 3. Current density distribution for the circular coil positioned above the head model and tilted $20^{\circ}$. The RI in respect to the maximum for each case is shown on the bottom. Left: realistic head layers surrounded by air. Middle and right: the head is surrounded by conducting liquid. The right image, in addition, shows all layers with identical conductivities $(\sigma)$. The absence of surface effect is notorious, as expected.
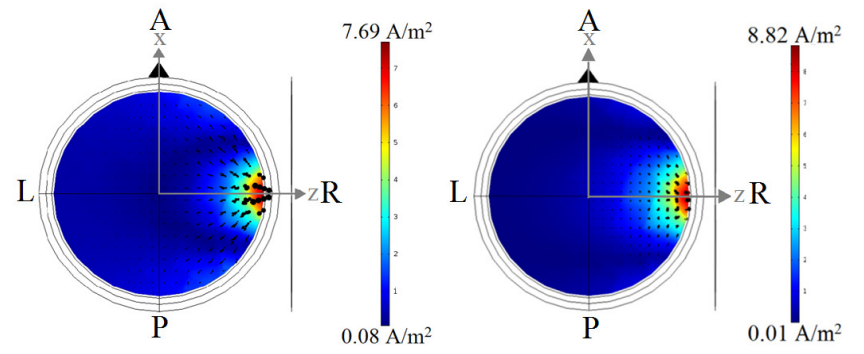

Figure 4. Axial views of the current density distributions for the figureeight coil. Left: head surrounded by air. Right: Head and coil immersed in conducting liquid, leading to not significantly larger RI at the center. 
eight coil is studied for a system surrounded by air (left, $0.64 \% \mathrm{RI}$ at the center) and a system immersed in conducting liquid (right, $5.68 \% \mathrm{RI}$ at the center).

\section{A. Mitigating Surface Effect: Example with Simple Coil}

The induced electrical field decreases rapidly with depth because the magnetic field due to the current in the coil also decreases rapidly with distance from the coil windings [17]. The decrease of the electric field with depth is enhanced by the surface charge that accumulates on the surface discontinuities [17]. The problem of surface discontinuities can be attenuated or solved by using a conducting liquid surrounding the stimulator coils and the spherical head model $[15,16]$. A possible solution for the implementation of such a conducting liquid is a saline solution [18].

Fig. 3 shows three simulations where the behavior of the minimum current density through brain tissue can be seen. When the head model is surrounded by air (left) or conducting liquid (middle) the minimum of current density shifts from the center to the anterior region, respectively. If the layers have equal conductivities (i.e. without discontinuities), the line with the minimum does not trespass the brain at all, as expected. This fact proves that the surface effect is due to surface discontinuities. In addition, using the conducting liquid mitigates surface effects that reduce brain penetration power in TMS. Therefore this concept seems to allow new insights for deep-brain TMS systems as well as its application in the treatment of several disorders.

\section{B. Results with Figure-Eight Coil}

In this section the performance of the figure-eight coil is studied. For this purpose the coil was positioned on the right side of the spherical head model in order to stimulate the right hemisphere (Fig. 4). Due to its high focality, the presence of the conducting liquid surrounding the system and the spherical head model do not have a substantial effect on the current density distribution. Nevertheless, the current density is always superior in average by about $33.2 \%$ when the conducting liquid scenario is taken into account. At the center of the brain this maximum difference reaches $87.7 \%$ (10 $\mathrm{cm}$ penetration depth).

\section{AN OPTIMIZED ORTHOGONAL CONFIGURATION}

The orthogonal configuration analyzed in this study results from previous collaborative works $[15,16]$. Here, a new extremely detailed system optimization has been achieved which resulted in a further increase of the effective penetration power by $10 \%$. In addition, system volume was decreased by $60 \%$. As shown in Fig.5, this optimized orthogonal configuration consists of five rectangular coils placed perpendicular to each other, except for one, slightly smaller, positioned within one of the larger ones. The whole assembly is immersed in conducting liquid together with part of the head of the patient, with the torso being involved by air only. The thresholds for heart fibrillation and magnetophosphenes (i.e. flashes of light) $[15,16]$ are again not reached by a high safety margin.
The aforementioned surface charge accumulation may be prevented if the surface electric discontinuity is minimized by substituting air as the exterior medium by a conducting liquid [19]. In addition, the transient current density induced in such external conducting liquid induces itself the biocurrents that may be observed in the brain. Indeed the values verified in the conducting liquid vary between 200 and $2700 \mathrm{~A} / \mathrm{m}^{2}$, a factor 10 to 100 larger than that verified in the skin. The latter are, in turn, approximately a factor 3 larger than those observed in the brain. The much larger current density in the conducting liquid proves that current induction in the brain due to skin current is negligible. In addition, the current direction in the conducting liquid is opposite to that in the coil and in the brain.

\section{A. Results of Whole-Brain Stimulation}

Fig. 6 shows the volume, coronal, axial, and sagittal views obtained of the head model stimulated with the orthogonal configuration in analysis in this work. It can be seen that the $\mathrm{RI}$ at the center of the head is 58\% (4.5\% RI if the assembly is surrounded by air). This value surpasses to the best of our knowledge the state of the art for deep-brain and whole-brain TMS, potentially opening new possibilities for the clinical use of TMS.

\section{B. Results of Half-Hemisphere Stimulation}

Emerging TMS theories state the existence of clinical benefit if only one brain hemisphere is either stimulated or inhibited. Examples under study include stroke, obsessive compulsive disorder, and depression, among others. In an attempt to provide a solution to this scenario the simulation with results presented in Fig. 7 was carried out. In order to achieve it, the current in the coil near the left hemisphere was switched off. It can be seen that mainly the right hemisphere contains TMS current density. In addition, the stimulation of the whole hemisphere is accomplished, with significant values being reached even for deep brain depths (38.6\% RI at the center).

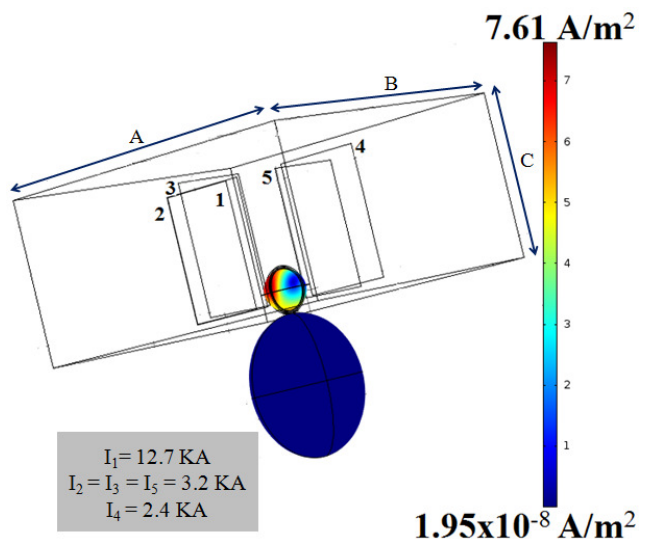

Figure 5. Three-dimensional view and main characteristics of the optimized orthogonal configuration studied here. The number of coils, their current values (termed $I_{1}-I_{5}$ ), together with the minimum and maximum values of current density achieved are shown. 

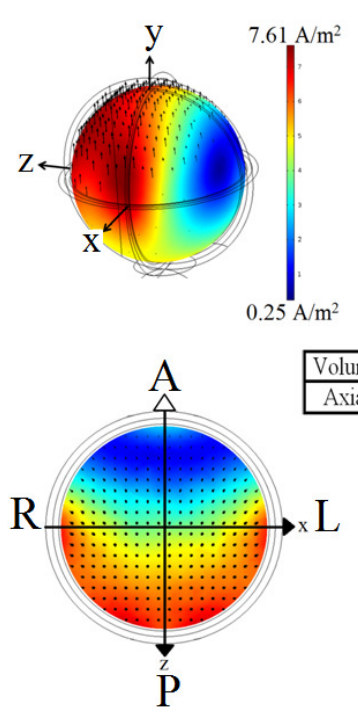

Figure 6. Current density distributions for the optimized orthogonal configuration stimulating the whole-brain. The coils and part of the head sphere are immersed in conducting liquid.
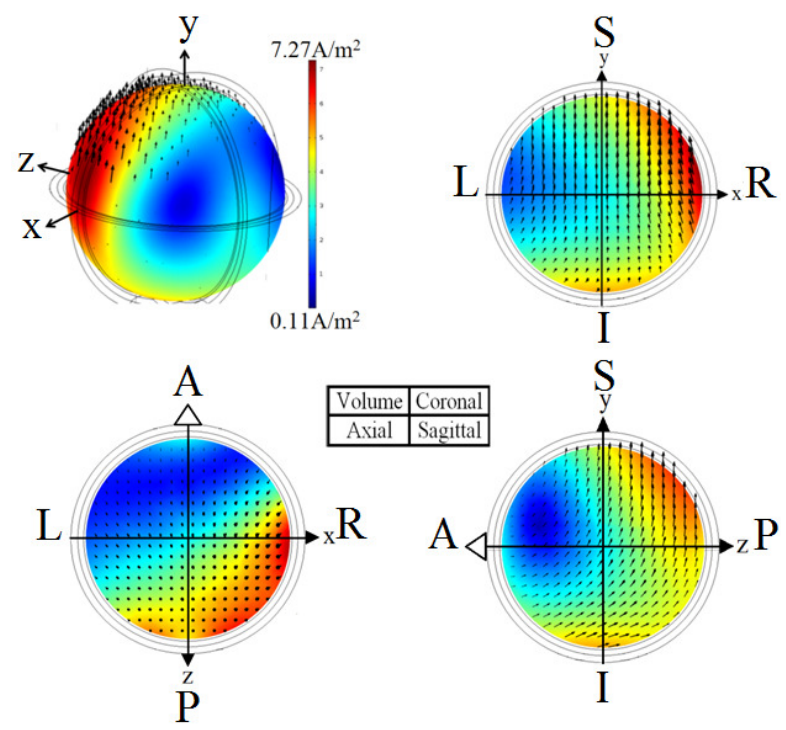

Figure 7. Current density distributions for the optimized orthogonal configuration stimulating mainly the right hemisphere, which could be further tilted upon need. The coils and part of the head sphere are immersed in conducting liquid.

\section{CONCLUSIONS}

$\mathrm{COMSOL}^{\circledR}$-based simulations show that the optimized orthogonal configuration immersed in conducting liquid under study in this work allows for a penetration power of $58 \%$ at a brain depth of $10 \mathrm{~cm}$. At $7 \mathrm{~cm}$ depth, the relative induction is $31 \%$ higher in respect to values published for the $\mathrm{H}$-coil. This work shows an increase of $10 \%$ in RI in respect to previous collaborative work, with in addition a decrease of $60 \%$ in the whole system volume.

We believe the results obtained in this work may allow for stimulation of deeper and whole-brain structures, therefore potentially contributing to new insights in clinical TMS.

\section{ACKNOWLEDGMENT}

The authors greatly acknowledge Prof. Pedro Cavaleiro Miranda, from the University of Lisbon, for important discussions, sharing of ideas and work suggestions.

\section{REFERENCES}

[1] M. Koenigs, J. Grafman, "The functional neuroanatomy of depression: distinct roles for ventromedial and dorsolateral prefrontal cortex" Behavioural Brain Research, vol. 201, pp. 239-43, 2009

[2] J. Downar, Z. J. Daskalakis, "New targets for rTMS in depression: a review of convergent evidence" Brain Stimulation, vol. 6, pp. 231-40, 2013

[3] R. Prinkryl, et al., "A detailed analysis of the effect of repetitive transcranial magnetic stimulation on negative symptoms of schizophrenia: a double-blind trial" Schizophrenia Research, vol. 149, pp.167-73, 2013

[4] A. Yadollahpour, et al., "Investigating the anticonvulsant effects of repetitive transcranial magnetic stimulation on perforant path kindling model in rats", Zahedan J of Research in Med Sci, pp. 29-33, 2013

[5] L. M. M. Chacón, et al., "Transcranial magnetic stimulation and refractory partial epilepsy" In Pharmacoresistance in Epilepsy: From Genes and Molecules to Promising Therapies, pp. 265-89, 2013

[6] P. Limousin, I.Martinez-Torres, "Deep brain stimulation for Parkinson's disease" Neurotherapeutics: $J$ Am Soc Exp NeuroTherapeutics, vol. 5, pp. 309-19, 2008

[7] F. Spagnolo, et al., "Excitatory deep repetitive transcranial magnetic stimulation with $\mathrm{H}$-coil as add-on treatment of motor symptons in Parkinson's disease: an open label, pilot study" Brain Stimulation, pp. $1-4,2013$

[8] B.R. Webster, et al., "Noninvasive brain stimulation in stroke rehabilitation" J Am Soc Exp NeuroTherapeutics, vol. 3, pp. 474-81, 2006

[9] R. Chieffo, et al., "Excitatory deep transcranial magnetic stimulation with H-coil over the right homologous Broca's region improves naming in chronic post-stroke aphasia" Neurorehabilitation and Neural Repair, pp. 1-8, 2013

[10] C. Civardi, et al., "Applications of transcranial magnetic stimulation in sleep medicine" Sleep Medicine Reviews, vol.13, pp. 35-46, 2009

[11] A. Tzabazis, et al., "Shaped magnetic field pulses by multi-coil repetitive transcranial magnetic stimulation (rTMS) differentially modulate anterior cingulate cortex responses and pain in volunteers and fibromyalgia patients" Molecular Pain, vol.3, pp. 1-9, 2013

[12] R. Nardone, et al., "Effect of transcranial brain stimulation for the treatment of Alzheimer disease: a review" Int J Alzheimer's Disease, 2012

[13] W. Paulus, et al., "Transcranial electric and magnetic stimulation: techniques and paradigms" In Brain Stimulation: Handbook of Clinical Neurology, vol. 116 (3rd series), pp. 229-342, 2013

[14] T. Fadini et al., "H-coil: induced electric field properties and input/output curves on healthy volunteers, comparison with a standard figure-of-eight coil," Clin Neurophysiol vol. 120, pp. 1174-82, 2009

[15] H. Oliveira, et al., "Multiple coils in a conducting liquid for deep and whole-brain transcranial magnetic stimulation. I. Single-frequency excitation" Bioengineering (ENBENG), 2nd IEEE Portuguese Meeting, Coimbra, Portugal, 23-26 Feb. 2012

[16] M. D. Silva, et al., "Multiple coils in a conducting liquid for deep and whole-brain transcranial magnetic stimulation. II. Single-frequency excitation" Bioengineering (ENBENG), 2nd IEEE Portuguese Meeting, Coimbra, Portugal, 23-26 Feb. 2012

[17] P. C. Miranda, "Physics effects of transcranial brain stimulation" In Brain Stimulation: Handbook of Clinical Neurology, vol. 116 (3rd series), pp. 353-366, 2013

[18] H. Simões, et al., "Experimental demonstration of induction by means of a transcranial magnetic stimulator coil immersed in a conducting liquid" Bioengineering (ENBENG), 3rd IEEE Portuguese Meeting, University of Minho, Portugal, 20-22 Feb. 2013

[19] R. Carbunaru and D.M. Durand, "Toroidal coil models for transcutaneous magnetic simulation of nerves" IEEE Trans Biomed Eng, vol. 48, no. 4, pp. 434-41, 2001 